\title{
Improved method for the estimation of plasma ammonia by ion exchange
}

\author{
J. C. B. FENTON AND A. H. WILLIAMS \\ From the Department of Chemical Pathology, St. Bartholomew's Hospital
}

SYNOPSIS Care in the preparation and preservation of reagents, together with certain modifications in apparatus and technique, have eliminated the difficulties from plasma ammonia analysis. Ammonia contamination from the laboratory atmosphere has not proved an especial hazard, and no major.. setback has been encountered in the analysis of several thousand specimens over the last few years. The method has been found particularly suited to the performance of the occasional estimation, since, once prepared, all reagents are stable for a considerable time.

The estimation of ammonia in blood has long been considered a difficult analysis and it has been stated that every investigation on the metabolism of ammonia is admittedly handicapped by the problem of methodology (Perea and Nelson, 1964). The commonly used methods of microdiffusion analysis are not well suited to the estimation of small quantities of ammonia in blood and to achieve the required sensitivity a most careful technique is needed to stretch the capacity of these methods to near their working limit.

Although methods for the estimation of plasma ammonia by ion-exchange have been proposed for several years (Fenton, 1960, 1962; Dienst, 1961) it does not seem widely recognized that methods based on this principle offer advantages in reproducibility and sensitivity not readily attainable by the microdiffusion technique.

In an earlier communication a method was described for the estimation of plasma ammonia by ion exchange (Fenton, 1962), and in view of the long history of difficulty with blood ammonia analysis, special precautions were taken to eliminate those sources of artefact which were considered to be important hazards. This method has now been in routine daily use for several years and with the experience gained during this time, it has been found possible to simplify the technique and make improvements to both reagents and apparatus.

The purpose of this account is to describe the modified method which is now used and to mention precautions which are necessary to obtain reliable results.

Received for publication 30 May 1967.

\section{MATERIALS AND APPARATUS}

It is essential that all distilled water is passed through a $\overrightarrow{\mathscr{C}}$ de-ionizing column to remove traces of ammonia and $\propto$ some metals.

HEPARIN Dried heparin powder, 100,000 i.u. (Evans Medical Ltd.) is dissolved in $4 \mathrm{ml}$. water. The somewhat viscid solution is passed down one of the ion exchange $\frac{2}{8}$ columns which are used for plasma ammonia analysis. $\varrho$ The ammonia-free effluent of heparin is collected and a $\vec{\overrightarrow{ }}$ further $1 \mathrm{ml}$. water is added to the column to displace the residuum. The final volume is made up to $5 \mathrm{ml}$.

BLOOD COLLECTING TUBES The heparin solution is dispensed into $10 \mathrm{ml}$. polystyrene disposable tubes, each tube receiving a total of 400 i.u. Other anticoagulants are less 0 satisfactory.

ION EXCHANGE RESIN (AMBERLITE C.G. 120) 100 to 200 MESH The resin is supplied as a dry powder and contains soluble impurities. The stated mesh size is only approximate and $\frac{9}{3}$ it is necessary therefore to grade and clean the resin as follows.

The resin is wet washed with tap water through a test $\mathrm{N}$ sieve, 100 mesh $(150 \mu$ aperture) and only particles. retained on the mesh are suitable for further use. The $N$ graded resin is treated in bulk in a large glass column and $N$ the following solutions are run through the resin bed in $\omega$ sequence. The volumes refer to the volume of the resin? bed.

Concentrated hydrochloric acid, 1 volume, and without $\bar{\varnothing}$ further delay once the acid has passed, distilled water, $1 \stackrel{\oplus}{\rightarrow}$ volume; $2 \mathrm{M}$ sodium chloride solution, 2 volumes; $0.05 \mathrm{M}$ T E.D.T.A., $p \mathrm{H} 7$ to 8,1 volume; $0 \cdot 1 \mathrm{~N}$ sodium hydroxide, 2 응 volumes; distilled water, 1 volume.

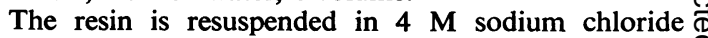
solution (ammonium free) and concentrated sulphuric 
acid is added drop by drop until the sodium chloride solution, which is in ionic equilibrium with the resin, maintains a neutral reaction. It is necessary to shake or stir the resin during this stage of neutralization but excessive agitation should be avoided since fracture and wear of the resin particles may produce an undesirable amount of 'fines'. The resin is stored at room temperature in $4 \mathrm{M}$ sodium chloride solution. An improvement in the blank value of the resin occurs on storage and it is an advantage to change the sodium chloride solution once after an interval of one month.

ION EXCHANGE COLUMNS Polystyrene plastic columns are made up from parts of disposable plastic tubes and syringes which are currently available. The necessary components for a suitable column are illustrated in Figure 1. These are a $10 \mathrm{ml}$. stoppered polystyrene disposable tube and a $2 \mathrm{ml}$. polystyrene disposable syringe.

The principle in the use of these columns is that the polystyrene tube acts as a reservoir; the hollow plunger of the syringe forms a column of the correct dimensions and the male needle fitting at the distal end of the syringe barrel is used to close the lower end of the column. The polystyrene components are best cut on a length of electrically heated resistance wire and the cut surfaces are then ground to a smooth finish on a stone to which has been added a little scouring powder and water. The prepared components are glued together with an adhesive of chloroform containing a little dissolved polystyrene.

A disc of woven nylon material is glued to the lower end of the column to act as a sieve support for the resin particles. Discs of nylon are readily cut by stretching woven material over a sheet of Perspex and using a hot cork borer as a punch.

The rate of flow of fluid down the column is controlled by the fitting of a hypodermic needle to the lower end of the column and a size 20 needle will provide a suitable restriction.

PREPARATION OF COLUMNS Plastic columns are charged with sufficient resin to make a $3 \times 0.7 \mathrm{~cm}$. bed of resin and the whole column is then filled with ammonium-free $4 \mathrm{M}$ sodium chloride solution. The columns are stoppered at the upper and lower ends with plastic stoppers and may then be stored indefinitely. The columns are ready for use at a moment's notice, when it is only necessary to drain off the sodium chloride solution, and wash with a few millilitres of fresh $4 \mathrm{M}$ sodium chloride solution followed by distilled water until free of excess saline.

The columns are held in a spring clip stand during analytical use and a stand of convenient design is illustrated in Figure 2.

SODIUM CHLORIDE SOLUTION, 4 M A.R. Sodium chloride, $500 \mathrm{~g}$., and $4 \mathrm{ml}$. $\mathrm{N}$ sodium hydroxide solution is added to $1,500 \mathrm{ml}$. distilled water. The solution is boiled until crystals of sodium chloride begin to separate out and is then allowed to cool. The solution of saturated alkaline sodium chloride is stored as a stock solution from which ammonium-free $4 \mathrm{M}$ sodium chloride is prepared as required; $500 \mathrm{ml}$. of the stock solution is diluted to $675 \mathrm{ml}$. with distilled water and after the addition of a

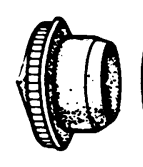

1

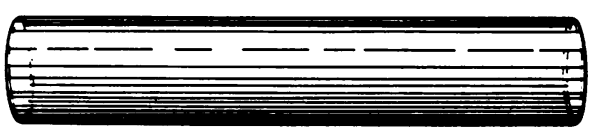

2

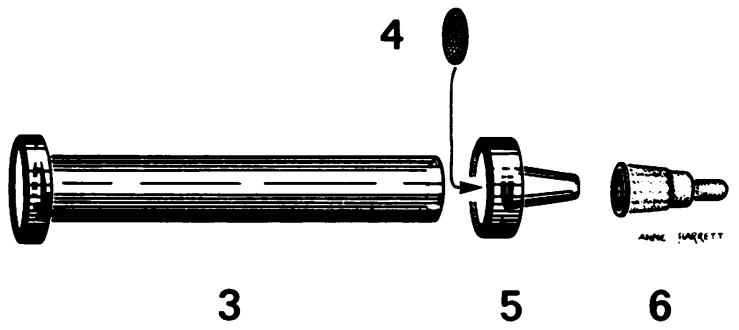

FIG. 1. The components of plastic ion exchange column. 1 and 2 Disposable plastic tube and stopper (Teklab, Hockley, Essex). 3 and 5 Plunger and lower end syringe barrel of $2 \mathrm{ml}$. plastic syringe (Shrimpton Steriseal Ltd., Redditch). 4 Disc of woven nylon. 6 Luer cap (Johnson's Ethical Plastics, Slough).

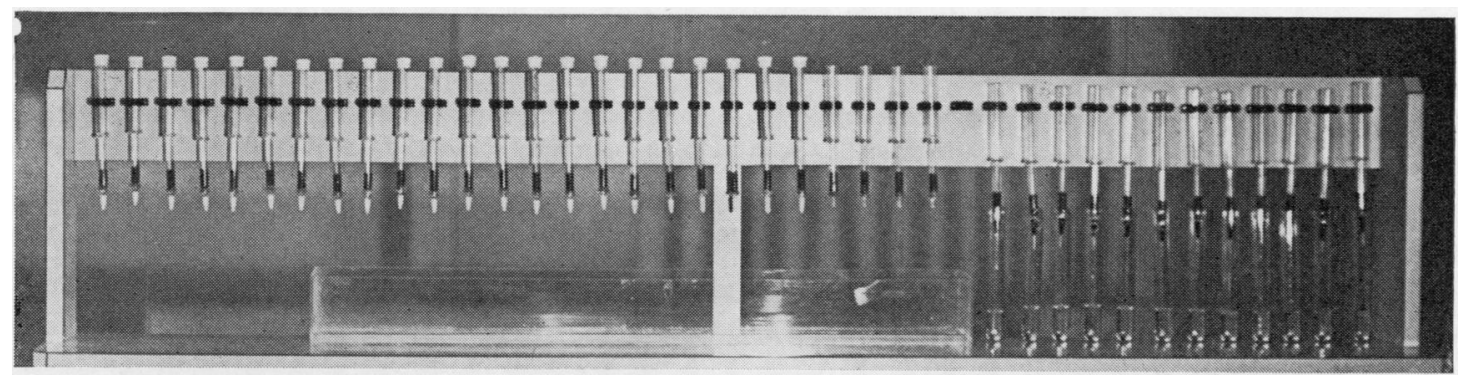

FIG. 2. Plastic columns in use on a spring clip stand. 
minimal amount of phenol red indicator, the solution is brought to neutrality by the addition of dilute sulphuric acid.

An occasional batch of A.R. sodium chloride has been found unsatisfactory on account of the presence of trace metals, which cause a depression of the indophenol blue colour reaction. If a neutral solution of sodium chloride is stored in a bottle containing dithizone dissolved in chloroform, interfering metals are removed. The sodium chloride solution is washed free of dithizone by shaking with fresh chloroform and the solution then rendered ammonia free as before.

DILUTING FLUID (0.2\% NONIDET AND $0.16 \mathrm{M}$ SODIUM CHLORIDE SOLUTION) The non-ionic detergent, Nonidet, for this solution is rendered ammonia-free by filtering a $5 \% \mathrm{w} / \mathrm{v}$ aqueous solution of Nonidet P.42 (British Drug Houses Ltd.) through an approximately $1 \times 4 \mathrm{~cm}$. column of Amberlite C.G. $120(\mathrm{Na})$ contained within the stem of a filter funnel which is plugged with glass wool at the lower end.

A stock solution of diluting fluid is prepared by mixing equal parts of $5 \%$ Nonidet solution with $4 \mathrm{M}$ ammoniafree sodium chloride solution. The components of this solution only remain miscible when stored cold in a refrigerator.

The working solution of diluting fluid is made by diluting $8 \mathrm{ml}$. of the stock solution to $100 \mathrm{ml}$. with distilled water. As this dilute solution may readily become contaminated, only small quantities are prepared at any one time. This dilute solution is stored in a refrigerator.

REAGENTS FOR THE INDOPHENOL BLUE REACTION Sodium hydroxide solution, $6.5 \mathrm{M}$, phenol reagent, and sodium nitroprusside solution are prepared as previously described (Fenton. 1962). The sodium nitroprusside solution and phenol reagent are stored in a refrigerator and no deterioration in the performance of these reagents has been noted after several years' storage.

ALKaline Phenol Reagent The approximate composition of this reagent is $10 \mathrm{ml}$. phenol reagent, $10 \mathrm{ml}$. sodium hydroxide solution, $6.5 \mathrm{M}$, and $0.2 \mathrm{ml}$. sodium nitroprusside solution. The total volume is then made up to $50 \mathrm{ml}$. with distilled water. However, each worker must determine for himself the optimal proportions of sodium hydroxide and phenol reagent, since some variation is inevitable between different batches of phenol.

To each of a series of $50 \mathrm{ml}$. volumetric flasks is added $10 \mathrm{ml}$. phenol reagent and $20 \mathrm{ml}$. distilled water. To each flask is then added increasing volumes of $6.5 \mathrm{M}$ sodium hydroxide solution, the volumes varying between $9 \mathrm{ml}$. and $11 \mathrm{ml}$. The contents of each flask are mixed, nitroprusside catalyst is added, and the volume made up with distilled water. The alkaline phenol reagent in each flask is used in turn to produce the indophenol blue reaction on a standard solution of ammonia as used in the actual method. When the resultant colours are compared, the proportion of alkali and phenol which gives the maximum colour development is chosen for subsequent use.

In the preparation of alkaline phenol reagent for use in an actual determination, $10 \mathrm{ml}$. phenol and the predeter- mined volume of $6.5 \mathrm{M}$ sodium hydroxide solution are made up to a volume of $50 \mathrm{ml}$. with distilled water. The 으․ mixture is cooled below $0{ }^{\circ} \mathrm{C}$. in a deep freeze and $0.2 \mathrm{ml}$. $\Rightarrow$ of $0.5 \%$ aqueous sodium nitroprusside added. The $\stackrel{\vec{P}}{+}$ reagent is now ready for use, but may be stored in a deep freeze for up to 14 days without deterioration.

SODIUM HYPOCHLORITE SOLUTION $0.35 \mathrm{~N}$ A solution of domestic 'bleach' is diluted with distilled water and the content of hypochlorite adjusted to approximately $0.35 \mathrm{~N}$ is in the manner previously described (Fenton, 1962).

The solution is stored in a brown glass bottle in a refrigerator and will then maintain a satisfactory strength $\vec{\omega}$ for up to nine months.

Cleaning OF Plastic COLUMNS AND glassware Plastic is columns, pipettes and other glassware are cleaned by $\overrightarrow{.}$ soaking in a solution of alkali and detergent (e.g., $\overrightarrow{\vec{A}}$ Haemosol).

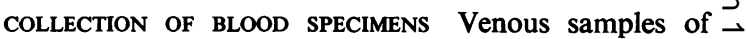
blood may be collected using brief venous stasis but open- $c$ ing and clenching the fist with exercise of the forearm muscles may induce falsely high values. The samples are placed in heparinized tubes and these should then reach $₹$ the laboratory within 30 minutes. Although cooling delays $\vec{\varphi}$ the formation of ammonia in shed blood, and tubes may be surrounded by crushed ice, very little change occurs within the first 30 minutes. The advantage of using an ice container seems to be that blood samples are treated with the careful urgency that these specimens require.

The blood samples are centrifuged and the plasma removed, care being taken not to remove plasma from the zone lying close to the buffy layer. The presence of haemolysis is undesirable because this may result in an occasional fictitious result. Clotted specimens of blood may also produce an elevation in the ammonia value.

If the sample of plasma cannot be analysed immediately it may be placed in a deep freeze at $-20^{\circ} \mathrm{C}$. and stored overnight without serious hazard to the final result.

\section{METHOD}

The plastic ion-exchange columns containing resin and $4 \mathrm{M}$ sodium chloride solution are shaken and inverted to 을 suspend the resin particles. The upper and lower stoppers are removed and the columns are placed in a vertical position in a spring clip stand. Care is taken to ensure that no air bubbles become trapped within the resin bed. $\sigma$

When the solution of sodium chloride has drained from $\mathrm{N}$ the columns a further $10 \mathrm{ml}$. of fresh sodium chloride solution is added and the effluent allowed to drip to waste. $\omega$

The columns are now given three washes of ammoniafree distilled water (approximately 2 to $3 \mathrm{ml}$. each wash) 0 in order to free the resin bed from excess saline. The lower $\frac{\bar{D}}{\mathbb{D}}$ stopper is replaced to close the column and $4 \mathrm{ml}$. of cold $\stackrel{\infty}{+}$ diluting fluid added. The diluting fluid is stored in a refrigerator and the bottle is only removed when $\bar{O}$ immediately required. The sample of plasma, $2 \mathrm{ml}$., is now added and after the lower stopper has been removed, the $\frac{O}{\Phi}$ contents of the column are allowed to drain through the $\varrho$ bed of resin and drip to waste. At this stage no needle is 
fitted to the column to restrict the flow rate, the time for passage of the fluid through the column being approximately 10 minutes.

The column is given several brief washes with distilled water in order to remove protein from the resin bed. The analysis may be stopped at this stage if desired; once the ammonium has been exchanged on to the resin the sample is stable at room temperature for several hours.

A size 20 hypodermic needle is washed through with water to displace air bubbles, and the needle then fitted to the lower end of the column. A test tube is positioned to collect the eluate and $4 \mathrm{ml}$. of $4 \mathrm{M}$ sodium chloride solution is added to the column. When the column has ceased to drip, $4 \mathrm{ml}$. distilled water is added and the collection of the eluate completed.

Ion exchange resin is discarded after use.

COLORIMETRIC ANALYSIS A blank is prepared using $4 \mathrm{ml}$. of $4 \mathrm{M}$ sodium chloride solution and $4 \mathrm{ml}$. distilled water. A suitable standard, equivalent to $200 \mu \mathrm{g}$. per $100 \mathrm{ml}$., consists of $4 \mathrm{ml}$. of $4 \mathrm{M}$ sodium chloride and $4 \mathrm{ml}$. dilute standard ammonium solution containing $1 \mu \mathrm{g}$. ammonium $\mathbf{N}$ per $\mathrm{ml}$.

Colorimetric analysis may be delayed some hours until a batch of samples has been collected. To each tube in turn is added $4 \mathrm{ml}$. alkaline phenol reagent and after mixing briefly, $1 \mathrm{ml}$. sodium hypochlorite solution is blown in from a pipette. The volume of hypochlorite solution is not critical but the solution should be added within 30 seconds after the addition of the alkaline phenol and the contents of the tube then mixed rapidly.

Although colour develops if the tubes are allowed to stand at room temperature, rather more colour results if the following variant in technique is used. The reagents are added in the manner described but the alkaline phenol reagent which has been stored in a deep freeze is added as a cold solution. The tubes are placed in a water bath at $37^{\circ} \mathrm{C}$. for 10 minutes and then allowed to stand at room temperature for a further $\mathbf{2 0}$ minutes before readings of the O.D. are made at $625 \mathrm{~m} \mu$. During colour development the tubes are placed in a shaded area of bench space and not exposed to bright daylight.

\section{RESULTS}

A common cause of difficulty in using this method of estimation in its original form was the deposition of protein over the surface of the ion exchange resin. As lipaemic samples of plasma tended to cause more trouble in this respect, the effect was tried of increasing the concentration of non-ionic detergent, which is used in the diluting fluid, and this has been of some benefit. The amount of heparin used in the anticoagulation of blood specimens has also been increased because some specimens appeared to deposit a fibrin-like protein over the resin surface. However, the most important cause of protein denaturation appeared to be some unknown property or impurity in the resin itself because a change in the method of cleaning and grading this material rather unexpectedly resulted in an improved performance.
When these modifications were employed, it was found that not only was there less protein absorbed on to the resin but also that little or no protein was subsequently eluted and the eluate was for practical purposes protein free. In the original procedure it had been necessary to precipitate protein from the eluate with aluminium hydroxide before the colorimetric reaction could be applied, but in view of the latter finding an experiment was made to see if this step could be omitted without detriment to the final result.

Duplicate samples of plasma were carried through the ion exchange method for ammonia as far as the stage of elution. The eluates from each pair of columns were then pooled, mixed, and divided into two halves. On one half of the eluate, the indophenol blue reaction for ammonia was carried out directly, while the other half was first treated with aluminium hydroxide before the colour reaction was applied. The results are shown in Table I, where it can be seen that there is little advantage to be gained by the use of aluminium hydroxide.

\section{TABLE I}

RESULTS WITH AND WITHOUT ALUMINIUM HYDROXIDE

\begin{tabular}{ccc} 
Specimen No. & $\begin{array}{c}\text { With } \mathrm{Al}(\mathrm{OH})_{3} \\
(\mu \mathrm{g} \mathrm{NH}, \mathrm{N} / 100 \mathrm{ml})\end{array}$ & $\begin{array}{c}\text { Without } \mathrm{Al}(\mathrm{OH})_{3} \\
\left(\mu \mathrm{g} \mathrm{NH} \mathrm{H}_{4}+\mathrm{N} / 100 \mathrm{ml}\right)\end{array}$ \\
\hline 1 & 39 & 38 \\
2 & 16 & 16 \\
3 & 15 & 16 \\
4 & 27 & 21 \\
5 & 32 & 28 \\
6 & 36 & 31 \\
7 & 92 & 90 \\
8 & 182 & 172 \\
9 & 49 & 45 \\
10 & 162 & 163 \\
11 & 176 & 183 \\
Mean & 75.09 & 73
\end{tabular}

It has been found that the ion exchange resin behaves as though it contained a source of nonexchangeable ammonia which is slowly released on storage. Although this is only small in amount it could nevertheless cause error if allowance is not made. The presence of this delayed release of ammonia is detected if a fresh column of resin is eluted with saline and then allowed to stand some hours. On re-eluting the column with saline a further small amount of ammonia will be found on colorimetric analysis. Fortunately, this release of ammonia does not continue indefinitely and if resin is stored in $4 \mathrm{M}$ saline for some weeks, the blank value declines to a negligible amount. Nevertheless the precaution is always taken of eluting columns with saline immediately before use.

The standard deviation for a single estimation based on a pooled variance of 96 replicate samples, 
was $5.92 \mu \mathrm{g}$. $\mathrm{NH}_{4}-\mathrm{N}$ per $100 \mathrm{ml}$. A recovery of 99 to $104 \%$ was obtained after the addition of ammonia to 28 samples of plasma in amounts calculated to provide supplements of 40 to $200 \mu \mathrm{g}$. $\mathbf{N H}_{4}-\mathrm{N}$ per $100 \mathrm{ml}$. Special care was taken not to disturb the upper layers of resin during the addition of $4 \mathrm{M}$ saline at the elution stage, because this has been found to be a cause of incomplete recovery.

The normal range of this method of estimation has been determined in 91 patients suffering from miscellaneous disorders, but excluding cases of liver disease and advanced heart or chest disease. In 93 estimations, the plasma ammonia was found to lie between 6 and $50 \mu \mathrm{g}$. $\mathrm{NH}_{4}-\mathrm{N}$ per $100 \mathrm{ml}$, with $25 \cdot 5$ as a mean value.

In a previous report, where the sample consisted solely of healthy young adults, the normal range was quoted as 3 to $27.5 \mu \mathrm{g}$. For the differential diagnosis of disease, it has become necessary to redefine the normal range among hospital patients, in whom advancing age or ill health tend to cause a small increase in plasma ammonia.

\section{DISCUSSION}

Modifications to the originally described technique became desirable, to increase the number of specimens which could be examined in a single experiment, to simplify certain steps in the procedure, and to reduce the labour in the preparation of apparatus.

Although any simple glass column can be used, the construction of plastic columns has been described because these have several advantages; they are less fragile and easier to clean and tend to retain the temperature of cool liquids added to them. Moreover their design makes it possible to store large numbers of columns charged with resin and ready for use at short notice.

The present method of analysis has been used in the performance of several thousand estimations of plasma ammonia and it has become quite clear that the successful application of this method depends more upon the careful preparation of reagents than upon the actual analytical technique. The prepara- tion of reagents involves some labour, but as most of these appear to be stable indefinitely under propero. conditions of storage, it is possible to determine the $\Rightarrow$ plasma ammonia under circumstances of occasionalos demand.

The hazard of contamination with ammonia is $\frac{\overline{\mathrm{D}}}{\mathrm{\sigma}}$ more apparent than real. No special room is necessary for this estimation and the occasional presence of ammonia vapour within the laboratory does notes seem to be a special cause of trouble. The best insurance against contamination is to adopt $\mathrm{a}_{-}$ defensive technique. Clean tubes are best kept in tins $\vec{\omega}$ with tight fitting lids; pipettes are not used dry, but? always rinsed through with distilled water immedio? ately before use. The inevitable presence of acidis fumes within the laboratory may cause glassware to become coated with a film of ammonium salts, but $\overrightarrow{-}$ trouble can be avoided by the use of dilute alkali foro cleaning purposes and by drying with heat.

There are now several published variants of the $\vec{c}$ indophenol blue reaction for ammonia. The particus lar reagent used in the present method is convenient because most of the components are stable for severaR years. Horn and Squire (1966) have suggested a use $\overrightarrow{-0}$ ful modification of this reaction and claim that the results are more reproducible. Their version repre sents an interesting advance in technique and we have been able to confirm that the reaction is also more sensitive. It has not been adopted for use at presento because an occasional specimen has given somewhato higher results for plasma ammonia and this is an aspect which requires further investigation.

We wish to thank Mr. M. P. Curwen of the Departmen of Medicine, Guy's Hospital Medical School, for statistical advice; Mrs. A. Barrett of the Department of Medical Illustration, St. Bartholomew's Hospital, for the preparation of figure 1 , and $\mathrm{Mr}$. P. Crocker of the Department of Pathology for figure 2.

\section{REFERENCES}

Dienst, S. G. (1961). J. Lab. clin. Med., 58, 149.

Fenton, J. C. B. (1960). Proc. Ass. clin. Biochemists, 1, 36. (1962). Clin. chim. Acta, 7, 163.

Horn, D. B., and Squire, C. R. (1966). Ibid., 14, 185.

Perea, V. D., and Nelson, R. S. (1964). Ibid., 9, 13. 